\title{
Chronic respiratory diseases are predictors of severe outcome in COVID-19 hospitalised patients: a nationwide study
}

\author{
Guillaume Beltramo $\mathbb{1}^{1,2,3,9}$, Jonathan Cottenet ${ }^{3,4,9}$, Anne-Sophie Mariet ${ }^{3,4,5,6}$, Marjolaine Georges ${ }^{1,3}$, \\ Lionel Piroth ${ }^{3,5,6,7}$, Pascale Tubert-Bitter ${ }^{8}$, Philippe Bonniaud ${ }^{1,2,3,9}$ and Catherine Quantin ${ }^{3,4,5,6,8,9}$
}

${ }^{1}$ Reference Constitutive Center for Rare Lung Diseases, Dept of Pulmonary Medicine and Intensive Care Unit, University Hospital, Dijon, France. ${ }^{2}$ INSERM LNC-UMR 1231, Dijon, France. ${ }^{3}$ University of Bourgogne-Franche-Comté, Dijon, France. ${ }^{4}$ Biostatistics and Bioinformatics (DIM), Dijon University Hospital, Dijon, France. ${ }^{5}$ INSERM, CIC 1432, Dijon, France. ${ }^{6}$ Clinical Epidemiology/Clinical Trials Unit, Clinical Investigation Center, Dijon University Hospital, Dijon, France. ${ }^{7}$ Infectious Diseases Dept, Dijon University Hospital, Dijon, France. ${ }^{8}$ Université Paris-Saclay, UVSQ, Université Paris-Sud, Inserm, High-Dimensional Biostatistics for Drug Safety and Genomics, CESP, Villejuif, France. ${ }^{9}$ These authors contributed equally to this work.

Corresponding author: Philippe Bonniaud (philippe.bonniaud@chu-dijon.fr)

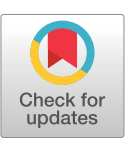

Copyright @The authors 2021.

This version is distributed under the terms of the Creative Commons Attribution Non-Commercial Licence 4.0. For commercial reproduction rights and permissions contact permissions@ersnet.org

This article has supplementary material available from erj.ersjournals.com

Received: 10 Dec 2020 Accepted: 1 May 2021

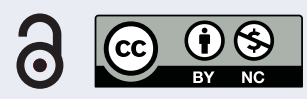

Shareable abstract (@ERSpublications)

There was a higher risk of developing severe COVID-19 and a higher mortality rate among patients with chronic respiratory diseases. This study suggests that these patients should have priority access to SARS-CoV-2 vaccination. https://bit.ly/3bcp2HC

Cite this article as: Beltramo G, Cottenet J, Mariet A-S, et al. Chronic respiratory diseases are predictors of severe outcome in COVID-19 hospitalised patients: a nationwide study. Eur Respir J 2021; 58: 2004474 [DOI: 10.1183/13993003.04474-2020].

\section{Abstract}

Background Influenza epidemics were initially considered to be a suitable model for the COVID-19 epidemic, but there is a lack of data concerning patients with chronic respiratory diseases (CRDs), who were supposed to be at risk of severe forms of COVID-19.

Methods This nationwide retrospective cohort study describes patients with prior lung disease hospitalised for COVID-19 (March-April 2020) or influenza (2018-2019 influenza outbreak). We compared the resulting pulmonary complications, need for intensive care and in-hospital mortality depending on respiratory history and virus.

Results In the 89530 COVID-19 cases, $16.03 \%$ had at least one CRD, which was significantly less frequently than in the 45819 seasonal influenza patients. Patients suffering from chronic respiratory failure, chronic obstructive pulmonary disease, asthma, cystic fibrosis and pulmonary hypertension were underrepresented, contrary to those with lung cancer, sleep apnoea, emphysema and interstitial lung diseases. COVID-19 patients with CRDs developed significantly more ventilator-associated pneumonia and pulmonary embolism than influenza patients. They needed intensive care significantly more often and had a higher mortality rate (except for asthma) when compared with patients with COVID-19 but without CRDs or patients with influenza.

Conclusions Patients with prior respiratory diseases were globally less likely to be hospitalised for COVID-19 than for influenza, but were at higher risk of developing severe COVID-19 and had a higher mortality rate compared with influenza patients and patients without a history of respiratory illness.

\section{Introduction}

On 11 March 2020 a global pandemic caused by a new coronavirus (severe acute respiratory syndrome coronavirus 2 (SARS-CoV-2)) was declared. The virus, which was first identified in December 2019, is responsible for coronavirus disease (COVID-19), a new disease with a predominant respiratory tropism.

Patients suffering from chronic respiratory diseases (CRDs), such as chronic respiratory failure (CRF), asthma, chronic obstructive pulmonary disease (COPD), interstitial lung diseases (ILDs), pulmonary hypertension, sarcoidosis or cystic fibrosis (CF), were immediately considered to be at risk of severe forms of COVID-19 [1]. Indeed, COVID-19 is responsible for various respiratory symptoms from cough with dyspnoea to acute respiratory distress syndrome (ARDS) in its most severe presentation [2, 3]. 
In parallel, it has been shown that COVID-19 patients have an increased risk of venous thromboembolic disease [4]. There is concern that the respiratory complications of COVID-19 could be deleterious in patients with prior CRDs. However, clinical experience and published data covering the outbreak period have suggested that there was a surprisingly low proportion of patients with CRDs among patients with COVID-19, especially in its most severe forms $[1,5,6]$.

Other viral respiratory diseases, and in particular influenza, are well known for their ability to induce severe respiratory complications [7], including ARDS, mainly in frail elderly people and in patients with severe comorbidities (obesity, diabetes, or heart or respiratory failure) [8]. Thus, the first Chinese and Italian epidemiological data, both on the mode of transmission and the respiratory tropism of SARS-CoV-2, initially led to a comparison of the pandemic to influenza epidemics in terms of spread and mortality [5]. This also explains the early recommendations for the prevention of the spread of SARS-CoV-2 infection, which were heavily based on knowledge acquired through the management of influenza epidemics. Existing knowledge of influenza was also used to define populations at risk of severe disease, particularly individuals with CRDs.

In a previous study by our group including 89530 in patients with COVID-19 identified over a 2-month period and 45819 seasonal influenza inpatients identified over a 3-month period, we found that patients diagnosed with COVID-19 were significantly less likely to have a history of CRDs than patients hospitalised for influenza [9].

Using the French national hospital database, the aim of our study was to describe and compare CRDs in hospitalised patients suffering from COVID-19 or influenza (2018-2019 season), and to describe and compare respiratory complications for COVID-19 patients with CRDs with COVID-19 patients without CRDs and with influenza patients.

\section{Methods}

\section{Database}

A retrospective cohort study was conducted using the national Programme de Médicalisation des Systèmes d'Information (PMSI) database, which is designed to include discharge summaries for all inpatient admissions to public and private hospitals in France. Diagnoses identified during the hospital stay are coded according to the International Statistical Classification of Diseases and Related Health Problems, 10th Edition (ICD-10) and procedures performed during hospitalisation are coded according to the French Common Classification of Medical Procedures (CCAM).

\section{Study design and participants}

Using the methodology described in the study by РіRotн et al. [9], all patients hospitalised for COVID-19 from 1 March 2020 to 30 April 2020 were included and identified by the primary diagnoses, related diagnoses or associated diagnoses using ICD-10 codes U0710, U0711, U0712, U0714 or U0715, regardless of their age (i.e. 89530 COVID-19 patients). For the influenza cohort, all patients hospitalised during the 2018-2019 influenza outbreak period (admitted from 1 December 2018 to 28 February 2019) and identified by ICD-10 codes J09, J10 or J11 (as primary diagnoses, related diagnoses or associated diagnoses) were included, whatever their age (i.e. 45819 influenza patients).

For each inpatient stay, we extracted age, sex, transfer to the intensive care unit (ICU) and hospital death. We also identified all diagnoses recorded in the discharge abstracts for the included hospital stays (COVID-19 and influenza) related to CRDs (CRF, lung cancer, pulmonary hypertension, sleep apnoea, COPD, emphysema, asthma, pulmonary sarcoidosis, ILDs and CF with pulmonary manifestations) and respiratory complications (acute respiratory failure, pulmonary embolism, ventilator-acquired pneumonia (VAP), pneumocystis pneumonia, aspergillosis pneumonia, pleural empyema, lung abscess and pneumothorax). We considered that patients suffered from VAP if they presented a community-acquired pneumonia and had a ventilation procedure. We also collected data on obesity, diabetes, hypertension, heart failure and atherosclerotic heart disease. The ICD-10 and CCAM codes used are presented in supplementary table S1.

This study was approved by the French Ethics and Scientific Committee for Research, Studies and Evaluation in Health (CESREES; 9 June 2020) and the French Institute of Health Data (INDS; 15 June 2020, registration 1611357), and authorised by the French Data Protection Authority (CNIL; 3 July 2020, registration number DR-2020-250). 


\section{Statistical analysis}

We described and compared age, sex and all diagnoses related to CRDs in patients hospitalised for COVID-19 or influenza. We also compared these CRDs in COVID-19 and influenza patients, depending on age grouped into nine classes $(<18,18-30,31-40,41-50,51-60,61-70,71-80,81-90$ and $>90$ years).

For each CRD, we then described and compared the need for intensive care, in-hospital mortality and all diagnoses related to respiratory complications in the COVID-19 and seasonal influenza groups.

Next, we compared the need for intensive care, in-hospital mortality and all diagnoses related to respiratory complications in the COVID-19 group between patients with and without CRDs. COVID-19 patients with CRDs were considered as a whole and then separately for each CRD in the sensitivity analyses. In the first sensitivity analysis, we classified our CRD patients as a single category. When several CRD codes were listed in the patient's discharge abstract, we prioritised in order to retain only one, in the following order: CF with pulmonary manifestations, lung cancer, COPD, asthma, pulmonary sarcoidosis, ILDs, emphysema, sleep apnoea, pulmonary hypertension and CRF (we selected the first CRD in this list). The second sensitivity analysis, to overcome the difficulty in prioritising CRD, included patients with only one CRD.

All identified respiratory diseases or complications are accounted for in relation to the patient. In other words, if a patient had one of the conditions at least once during one of the stays for COVID-19 or for influenza, then the patient was considered to have had the condition. Categorical data are provided as frequencies (percentage). Continuous data are provided as mean with standard deviation and as median (interquartile range (IQR)). The different variables studied were compared using the Chi-squared test or Fisher's exact test for categorical data and the t-test or Mann-Whitney test for continuous data.

To estimate the risk of transfer to the ICU and the risk of in-hospital mortality in COVID-19 patients with or without CRDs, we performed three logistic regressions adjusting on obesity, diabetes, hypertension, heart failure, atherosclerotic heart disease, sex and age as a continuous variable: 1) a global model including CRDs overall, 2) we used the same model with CRDs as disjunctive variables using the prioritisation defined earlier (first sensitivity analysis) and 3) a model with CRDs as disjunctive variables for patients with only one CRD (second sensitivity analysis). For each of the models, patients without CRDs were considered as the reference. Adjusted odds ratios (aORs) with 95\% confidence intervals are given.

The statistical significance threshold was set to $\mathrm{p}<0.05$. All analyses were performed using SAS version 9.4 (SAS Institute, Cary, NC, USA).

\section{Results}

The characteristics of the patients suffering from CRDs among the 89530 COVID-19 patients and the 45 819 influenza patients and for each of the viral epidemics are detailed in table 1 . Among the patients suffering from COVID and seasonal influenza, 14351 (16.03\%) and 9131 (19.93\%), respectively, presented at least one CRD. Among COVID patients with CRDs, $80.91 \%$ of them had only one CRD. There were significantly fewer patients with a history of CRF, COPD, asthma, CF and pulmonary hypertension in the COVID-19 compared with the seasonal influenza cohort $(\mathrm{p}<0.0001$ for all). On the contrary, COVID-19 patients were more likely to have a diagnosis of lung cancer, sleep apnoea, emphysema and ILDs $(p<0.0001$ for all, except for lung cancer $p=0.0098)$. The proportion of pulmonary sarcoidosis did not significantly differ between the two groups. After stratification by age (supplementary table S2), lung cancer and emphysema were more often found among elderly COVID-19 patients (aged 81-90 and >70 years, respectively), whereas sleep apnoea was more often found in younger patients (aged 31-40 years). Pulmonary hypertension, COPD, asthma and CF were less frequent among COVID-19 patients regardless of age strata, except, interestingly, for asthma in patients aged $<18$ years.

Among the patients with CRDs, we described and compared disease progression and prognosis in the case of SARS-CoV-2 or influenza infection. For the respiratory complications in patients with CRDs stratified by disease (table 2 and supplementary table S3), we found that COVID-19 patients developed significantly more VAP than influenza patients. Regarding underlying CRDs, VAP was significantly more frequent in COVID-19 patients suffering from pulmonary hypertension, sleep apnoea, emphysema, asthma, pulmonary sarcoidosis and ILDs. However, VAP was significantly more frequent for influenza patients suffering from CRF or lung cancer. No difference was observed for COPD and CF. On the contrary, COVID-19 patients presented pulmonary embolism significantly more often than influenza patients, except for those with lung cancer or pulmonary sarcoidosis. In the COVID-19 group, patients diagnosed with sleep apnoea, asthma, pulmonary sarcoidosis and ILDs were significantly more likely to require intensive care than other patients 
TABLE 1 Respiratory history of patients hospitalised in France for COVID-19 (1 March 2020 to 30 April 2020) or seasonal influenza (1 December 2018 to 28 February 2019)

\begin{tabular}{|c|c|c|c|}
\hline & COVID-19 & 2018-2019 seasonal influenza & p-value \\
\hline Patients & 89530 & 45819 & \\
\hline Male & 47495 (53.05) & $22118(48.27)$ & $<0.0001$ \\
\hline Age & $65 \pm 20$ & $59 \pm 32$ & $<0.0001$ \\
\hline Obese $\left(\mathrm{BMI} \geqslant 30 \mathrm{~kg} \cdot \mathrm{m}^{-2}\right)$ & $8611(9.62)$ & $2491(5.44)$ & $<0.0001$ \\
\hline Diabetes & $17050(19.04)$ & $7352(16.05)$ & $<0.0001$ \\
\hline Hypertension & 29622 (33.09) & $12921(28.20)$ & $<0.0001$ \\
\hline Heart failure & $7134(7.97)$ & $6266(13.68)$ & $<0.0001$ \\
\hline CRF & $1433(1.60)$ & $1830(3.99)^{\#}$ & $<0.0001$ \\
\hline Sleep apnoea & $3581(4.00)^{\#}$ & $1443(3.15)$ & $<0.0001$ \\
\hline Pulmonary hypertension & $341(0.38)$ & $247(0.54)^{\#}$ & $<0.0001$ \\
\hline COPD & $4866(5.44)$ & $4637(10.12)^{\#}$ & $<0.0001$ \\
\hline Asthma & $3273(3.66)$ & $2230(4.87)^{\#}$ & $<0.0001$ \\
\hline CF with pulmonary manifestations & $20(0.02)$ & $79(0.17)^{\#}$ & $<0.0001$ \\
\hline Lung cancer & $977(1.09)^{\#}$ & $431(0.94)$ & 0.0098 \\
\hline Emphysema & $1426(1.59)^{\#}$ & $553(1.21)$ & $<0.0001$ \\
\hline Pulmonary sarcoidosis & $159(0.18)$ & $81(0.18)$ & 0.9732 \\
\hline ILDs & $1611(1.80)^{\#}$ & $471(1.03)$ & $<0.0001$ \\
\hline
\end{tabular}

(table 2). In contrast, in the influenza group, patients with CRF, lung cancer and COPD were significantly more often treated in the ICU. Concerning in-hospital mortality (table 2), patients with CRDs were significantly more likely to die in the COVID-19 group, except for patients with CF.

Next, we compared the occurrence of respiratory complications, need for intensive care and in-hospital mortality in patients suffering from COVID-19 with and without CRDs. We observed a significant increase in the occurrence of each complication (except for pneumocystis pneumonia), need for intensive care and in-hospital mortality in patients with CRDs (table 3). In multivariate analyses, the risk of both ICU care and in-hospital mortality was increased for COVID-19 patients with CRDs compared with COVID-19 patients without CRDs (aOR 1.34, 95\% CI 1.28-1.41; and aOR 1.19, 95\% CI 1.14-1.25; respectively). The first sensitivity analysis using CRD prioritisation (table 4) showed that COVID-19 patients with lung cancer, COPD, sleep apnoea, emphysema and ILDs had significantly more complications (for most complications) than COVID-19 patients without CRDs. With the exceptions of CF and pulmonary sarcoidosis, patients with CRDs had significantly more acute respiratory failure. With the exceptions of CF and CRF, patients with CRDs had significantly more VAP. Excluding CF, lung cancer and CRF, all other CRDs significantly increased the risk of need for intensive care (table 4 and crude odds ratios in table 5). Excluding CF, asthma and emphysema, all other CRDs significantly increased the risk of in-hospital mortality (table 4 and crude odds ratios in table 5). It should be noted that lung cancer reduced the risk of VAP and need for intensive care, and that asthma reduced the risk of in-hospital mortality. In multivariate analyses in COVID-19 patients (table 5), we still found that, compared with patients without CRDs, lung cancer reduced the risk of need for intensive care (aOR 0.77, 95\% CI 0.63-0.94), while the other CRDs increased it (except for CF and CRF). Concerning mortality, multivariate analysis (table 5) showed that sleep apnoea and pulmonary hypertension were no longer associated with the risk of death, while pulmonary sarcoidosis became associated with this risk (aOR 2.11, 95\% CI 1.36-3.26). Because of the relationship between sleep apnoea and obesity (36.5\% of obesity in sleep apnoea patients and $7.11 \%$ in patients without CRDs), we took into account this possible interaction in our model, but it did not influence our results. The other CRDs were still associated with the risk of in-hospital mortality (except for emphysema). This was particularly true for lung cancer, which multiplied the risk of death by more than 3.5 (aOR 3.67, 95\% CI 3.20-4.21).

The results of the second sensitivity analysis including COVID-19 patients with only one CRD were generally consistent with the previous results (supplementary tables S4 and S5). However, patients with COPD were no longer associated with the risk of need for intensive care and patients with ILDs were no longer associated with the risk of in-hospital mortality in multivariate analysis (supplementary table S5). 
TABLE 4 Respiratory morbidity, need for intensive care and mortality of patients hospitalised in France for COVID-19 (1 March 2020 to 30 April 2020): comparison between patients with and without chronic respiratory disease (CRD) (disjunctive variables using prioritisation ${ }^{\#)}$

\begin{tabular}{|c|c|c|c|c|c|c|c|c|c|}
\hline CF & $\begin{array}{l}\text { Lung } \\
\text { cancer }\end{array}$ & COPD & Asthma & $\begin{array}{l}\text { Pulmonary } \\
\text { sarcoidosis }\end{array}$ & ILDs & Emphysema & $\begin{array}{c}\text { Sleep } \\
\text { apnoea }\end{array}$ & $\mathrm{PH}$ & CRF \\
\hline
\end{tabular}

\begin{tabular}{|c|c|c|c|c|c|c|c|c|c|c|c|}
\hline Patients & $20(0.1)$ & $977(6.8)$ & $4682(32.6)$ & $2973(20.7)$ & $138(1.0)$ & $1385(9.7)$ & $794(5.5)$ & $2623(18.3)$ & $230(1.6)$ & $529(3.7)$ & \\
\hline $\begin{array}{l}\text { Pulmonary } \\
\text { embolism }\end{array}$ & $0(0)$ & $44(4.5)^{4}$ & $142(3.0)$ & $106(3.6)$ & $8(5.8)$ & $81(5.9)^{4}$ & $58(7.3)^{4}$ & $112(4.3)^{4}$ & $27(11.7)^{\natural}$ & $12(2.3)$ & 3.3 \\
\hline VAP & $1(5.0)$ & $49(5.0)^{+}$ & $445(9.5)^{9}$ & $323(10.9)^{\natural}$ & $26(18.8)^{4}$ & $277(20.0)^{\natural}$ & $132(16.6)^{4}$ & $517(19.7)^{\natural}$ & $33(14.4)^{4}$ & $39(7.4)$ & 7.9 \\
\hline $\begin{array}{l}\text { Pneumocystis } \\
\text { pneumonia }\end{array}$ & 0 & 0 & 0 & 0 & 0 & 0 & 0 & 0 & 0 & 0 & 0 \\
\hline $\begin{array}{l}\text { Aspergillosis } \\
\text { pneumonia }\end{array}$ & $1(5.0)^{\natural}$ & $1(0.1)$ & $32(0.7)^{4}$ & $15(0.5)^{4}$ & $0(0.0)$ & $13(0.9)^{4}$ & $6(0.8)^{4}$ & $8(0.3)$ & $1(0.5)$ & $3(0.6)$ & 0.2 \\
\hline Pleural empyema & $0(0)$ & $6(0.6)^{4}$ & $3(0.1)$ & $2(0.1)$ & $1(0.7)$ & $3(0.2)$ & $2(0.3)$ & $2(0.1)$ & $0(0)$ & $0(0)$ & 0.1 \\
\hline Lung abscess & $0(0)$ & $6(0.6)^{4}$ & $2(0.0)$ & $2(0.1)$ & $0(0)$ & $9(0.7)^{4}$ & $6(0.8)^{4}$ & $2(0.1)$ & $0(0)$ & $1(0.2)$ & 0.1 \\
\hline Pneumothorax & $1(5.0)$ & $10(1.0)^{4}$ & $25(0.5)^{9}$ & $13(0.4)$ & $1(0.7)$ & $23(1.7)^{\natural}$ & $26(3.3)^{q}$ & $19(0.7)^{\natural}$ & $2(0.9)$ & $3(0.6)$ & 0.3 \\
\hline ARF & $3(15.0)$ & $344(35.2)^{9}$ & $1845(39.4)^{4}$ & $853(28.7)^{\natural}$ & $43(31.2)$ & $632(45.6)^{4}$ & $297(37.4)^{4}$ & $1068(40.7)^{4}$ & $99(43.0)^{9}$ & $203(38.4)^{4}$ & 25.2 \\
\hline ICU & $2(10.0)$ & $117(12.0)^{+}$ & $960(20.5)^{4}$ & $570(19.2)^{\natural}$ & $47(34.1)^{9}$ & $453(32.7)^{4}$ & $213(26.8)^{9}$ & $851(32.4)^{4}$ & $59(25.7)^{9}$ & $91(17.2)$ & 14.9 \\
\hline In-hospital death & $0(0)$ & $402(41.2)^{4}$ & $1163(24.8)^{4}$ & $266(9.0)^{+}$ & $29(21.0)$ & $296(21.4)^{\bullet}$ & $147(18.5)$ & $466(17.8)^{4}$ & $64(27.8)^{4}$ & $152(28.7)^{\natural}$ & 16.1 \\
\hline
\end{tabular}

Data are presented as $\mathrm{n}(\%)$ or \%. CF: cystic fibrosis; COPD: chronic obstructive pulmonary disease; ILD: interstitial lung disease; PH: pulmonary hypertension; CRF: chronic respiratory failure; VAP: ventilator-associated pneumonia; ARF: acute respiratory failure; ICU: intensive care unit. "\#: each CRD patient was classified in only one single CRD category (when there were several CRD codes on the discharge abstract of a patient, we decided to prioritise in order to retain only one, using the following order: CF with pulmonary manifestations, lung cancer, COPD, asthma, pulmonary sarcoidosis, ILDs, emphysema, sleep apnoea, PH and CRF); ': significantly more frequent in the CRD group compared with the group with no CRD $(p<0.05) ;{ }^{+}$: significantly less frequent in the CRD group compared with the group with no CRD $(p<0.05)$.

over-represented among patients hospitalised for COVID-19 when compared with influenza. Compared with COVID-19 patients without CRDs, those with CRDs were more likely to need ICU care and to die. However, patients with CRF, lung cancer and COPD in the COVID-19 group were significantly less likely to be admitted to the ICU compared with the influenza group. Moreover, when comparing risk of requiring intensive care, we observed that patients with lung cancer were admitted less frequently than patients without

TABLE 5 Risk of requiring intensive care and mortality for patients hospitalised in France for COVID-19 (1 March 2020 to 30 April 2020) according to chronic respiratory disease (CRD) (disjunctive variables using prioritisation ${ }^{\#)}$

\begin{tabular}{|c|c|c|c|c|}
\hline & \multicolumn{2}{|r|}{ ICU } & \multicolumn{2}{|c|}{ Death } \\
\hline & OR $(95 \% \mathrm{Cl})$ & Adjusted OR $(95 \% \mathrm{Cl})$ & OR $(95 \% \mathrm{Cl})$ & Adjusted OR $(95 \% \mathrm{Cl})$ \\
\hline No CRD & 1 & 1 & 1 & 1 \\
\hline CF & $0.63(0.15-2.73)$ & $0.60(0.1-2.60)$ & & \\
\hline Lung cancer & $0.78(0.64-0.94)^{9}$ & $0.77(0.63-0.94)^{4}$ & $3.64(3.20-4.14)$ & $3.67(3.20-4.21)$ \\
\hline COPD & $1.47(1.37-1.58)$ & $1.16(1.07-1.26)$ & $1.72(1.61-1.84)$ & $1.14(1.06-1.22)$ \\
\hline Asthma & $1.35(1.23-1.48)$ & $1.23(1.12-1.36)$ & $0.51(0.45-0.58)^{9}$ & $0.82(0.71-0.94)^{9}$ \\
\hline Pulmonary sarcoidosis & $2.94(2.07-4.19)$ & $2.65(1.83-3.84)$ & $1.38(0.92-2.09)$ & $2.11(1.36-3.26)$ \\
\hline ILDs & $2.77(2.47-3.11)$ & $2.42(2.14-2.72)$ & $1.41(1.24-1.61)$ & $1.20(1.05-1.38)$ \\
\hline Emphysema & $2.09(1.78-2.45)$ & $1.83(1.56-2.16)$ & $1.18(0.99-1.42)$ & $1.01(0.83-1.22)$ \\
\hline Sleep apnoea & $2.74(2.52-2.98)$ & $1.39(1.27-1.53)$ & $1.12(1.02-1.25)$ & $0.95(0.85-1.06)$ \\
\hline Pulmonary hypertension & $1.97(1.46-2.65)$ & $1.73(1.27-2.37)$ & $2.01(1.50-2.68)$ & $1.24(0.91-1.67)$ \\
\hline CRF & $1.18(0.94-1.49)$ & $1.03(0.81-1.30)$ & $2.10(1.74-2.54)$ & $1.30(1.06-1.59)$ \\
\hline \multicolumn{5}{|c|}{$\begin{array}{l}\text { Odds ratios adjusted on obesity, diabetes, hypertension, heart failure, atherosclerotic heart disease, sex and } \\
\text { age as a continuous variable. ICU: intensive care unit; CF: cystic fibrosis; COPD: chronic obstructive pulmonary } \\
\text { disease; ILD: interstitial lung disease; CRF: chronic respiratory failure. \#: each CRD patient was classified in only } \\
\text { one single CRD category (when there were several CRD codes on the discharge abstract of a patient, we } \\
\text { decided to prioritise in order to retain only one, using the following order: CF with pulmonary manifestations, } \\
\text { lung cancer, COPD, asthma, pulmonary sarcoidosis, ILDs, emphysema, sleep apnoea, pulmonary hypertension } \\
\left.\text { and CRF); }{ }^{\uparrow} \text { : CRDs significantly less at risk ( } p<0.05\right) \text {. }\end{array}$} \\
\hline
\end{tabular}


CRDs, which may have been the result of ethical considerations during the crisis. However, all patients with a respiratory disease in the COVID-19 group had a higher mortality risk compared with the influenza group.

Obesity [10], diabetes [11], hypertension [12] and, especially, age [13] have been widely described as the main risk factors for developing severe forms of COVID-19. On the contrary, patients with respiratory diseases, in particular asthma and COPD, seem to be less likely to be severely affected [12, 14-17]. For example, two studies conducted in the New York City area, which was an early COVID-19 hotspot, found that COPD was a comorbid condition in only $5.4 \%$ of cases $[18,19]$, while it was found in $18 \%$ of patients hospitalised for influenza from 15 November 1999 to 15 April 2000 [20]. Regarding asthma (3.66\% in our national COVID-19 cohort), a low prevalence is also generally reported [21, 22], with some exceptions [23]. This is also the case with patients with CF, for whom the incidence of severe COVID-19 seems to be lower than in the general population [24, 25].

Our study adds data for COPD, asthma and CF, which were also less frequent in the COVID-19 group. This could be explained by several reasons. First, these fragile individuals may have been more careful and implemented more strict preventive measures, as already reported $[17,26]$. Second, the general lockdown applied during most of the study period led to a considerable decrease in air pollution [27], which is known to favour lung disease exacerbation [28], even in COVID-19 [29]. Third, the COVID-19 epidemic occurred later in the year than influenza and the weather was unusually warm. Since the cold is also described as influencing respiratory health, the effect of climate could be advocated here [30]. Fourth, COPD, asthma and CF are often treated by inhaled corticosteroids (ICSs). This treatment might have protected against COVID-19 since some in vitro models show that certain ICSs suppress SARS-CoV-2 replication and cytokine production [14, 31]. However, no study has confirmed this hypothesis and there is currently no evidence that ICS use protects against COVID-19 [26, 32, 33].

On the contrary, emphysema, lung cancer, ILDs and sleep apnoea were more frequent in patients hospitalised for COVID-19 than for influenza. The results for emphysema and lung cancer differed according to patient age. There were more patients with emphysema only among hospitalised COVID-19 patients aged $>70$ years. The same was observed for lung cancer, which was only significantly more present in elderly patients (aged 8190 years) in the COVID-19 group. No previous data support an increased risk of COVID-19 for these two conditions. The difference between emphysema and COPD seemed to be linked to the patient's profile. Indeed, only $30 \%$ of patients with emphysema had COPD and only 3.3\% had ILDs (combined pulmonary fibrosis and emphysema). These cases may correspond to smokers with emphysema but without obstructive syndrome. COVID-19 patients had significantly more emphysema in the oldest age groups; these were probably patients with emphysema without obstructive syndrome, for whom age seems to be the most important risk factor for developing COVID-19. Some publications underline the risk of severe COVID-19 and increased mortality risk in patients with ILDs [34]. However, Guiot et al. [35] found that only 1\% of patients with ILDs were hospitalised for COVID-19 (one in the ICU) among 401 patients in a single Belgian centre. Our results suggest the need to be cautious regarding ILD patients and the risk of SARS-CoV-2 infection.

The high prevalence of sleep apnoea syndrome in the population of patients hospitalised for COVID-19 could be explained by the fact that the risk factors for severe SARS-CoV-2 pneumonia (obesity, hypertension and diabetes, as previously described) are also well-known comorbidities of sleep apnoea [36]. Accounting for a potential interaction between obesity and sleep apnoea did not change our results for ICU and mortality risk. Moreover, obstructive sleep apnea has been identified as a risk factor for severe COVID-19 (including hospitalisation, ICU admission, mechanical ventilation or death [37]) and it may be an important predictor of poor outcomes in obese patients [38].

We identified several key points in our analysis of pulmonary complications and prognosis in patients hospitalised for COVID-19 with a history of respiratory disease. First, these patients developed significantly more VAP. Previous work reports that COVID-19 patients developed less respiratory bacterial co-infections than patient hospitalised for influenza [39]. The small number of patients with COVID-19 who developed respiratory bacterial co-infections made it possible (due to a lack of specific code for VAP and inconsistent ICU practices) to consider that community-acquired pneumonia crossed with codes for ventilation acts as VAP. Second, they developed significantly more pulmonary embolism than patients hospitalised for influenza and significantly more pulmonary complications than patients without CRDs hospitalised for COVID-19 [9]. Third, we found that patients hospitalised for COVID-19 and presenting CRDs were more likely to be admitted to the ICU, and mortality was increased compared with both patients hospitalised for influenza (except for lung cancer and COPD) and patients hospitalised for COVID-19 without CRDs. These data confirm that pulmonary diseases including CRF and asthma are associated with a risk of severe disease and mortality in SARS-CoV-2 infection, as indicated by national studies in the UK [31] and Sweden [40]. Nevertheless, while BLoom et al. 
[31] observed an increased mortality for patients with severe asthma and COVID-19, we observed a significant decrease in mortality compared with patients without CRDs. This decrease could be explained by protective effect of ICSs, as previously suggested by Вцоом et al. [31], and by the small proportion ( 10.0-10.8\% in BLoom et al. [31]) of severe asthma in our nationwide population. In COVID-19 patients with COPD (5.4\%), ICU admission was less frequent, but we also observed higher in-hospital mortality in the COVID-19 group than in the influenza group. These data are consistent with previously cited publications and several reviews $[41,42]$. In contrast to influenza infection, only few publications underline the role of CRDs in the development of severe forms of COVID-19 and even less so for hospital admission for COVID-19. This can be seen in a meta-analysis by ZHENG et al. [43], which reported that underlying respiratory disease had an OR of 5.15 for critical care or death. Regarding mortality, Khan et al. [44] found that the mortality risk in COVID-19 doubled in patients with respiratory disease [44].

We were unable to assess whether vaccination against influenza could explain the differences in morbidity and mortality in our study. Indeed, influenza vaccination, which is recommended for patients with CRDs, may have protected them from the most severe forms of influenza. Regardless, our data suggest that this category of patients should benefit from greater vigilance and be hospitalised at the slightest clinical sign of severe COVID-19. A more aggressive therapeutic approach may also be worth considering. Finally, our data suggest that patients with CRDs should have priority access to SARS-CoV-2 vaccination.

Our study has several limitations. First, there may have been testing biases between viruses because probably more patients were tested for COVID-19 than for influenza during the 2018-2019 season. Testing practices were also more standardised during the health crisis than during typical seasonal influenza epidemics. However, tests systematically done in the ambulatory setting for COVID-19 likely did not influence the number of hospitalised patients, particularly those admitted to the ICU. Second, the comparison between COVID-19 and seasonal influenza was done on two different years and the 2018-2019 influenza season is not necessarily representative of all seasonal influenzas, even if it was one of the most severe epidemics in the past 5 years in France. Although some COVID-19 patients may have had influenza at the same time, the influenza epidemic was ending at the start of the COVID-19 epidemic, limiting this possibility. Our study concerns only hospitalised patients and the characteristics of patients who died from COVID-19 outside of hospital may not reflect those described in our study. For respiratory history, certain CRDs may have been diagnosed during hospitalisation, potentially inducing a bias regarding the increased number of these diseases. For COVID-19 patients, this bias could include lung cancer, emphysema and ILDs due to the more frequent use of computed tomography pulmonary angiography for COVID-19 diagnosis, and because of the risk of pulmonary embolism, as previously reported [45]. Another possible limitation is misclassification-related or underdetection-related bias for comorbidities even if coding quality is checked by medical information professionals in each hospital to correct the diagnoses and to increase the recorded comorbidity level. These potential biases may concern comorbidities which were only identified during the stays for COVID-19 or for influenza, seeing as we cannot always distinguish between acute and chronic conditions. However, this misclassification bias is likely to be nondifferential for the majority of comorbidities, except for ILDs, for which the same code is used for pre-existing ILDs and for ILDs linked to the fibrosing ARDS complication. Finally, some relevant variables were not available such as treatments and tobacco consumption, which is not always coded in the discharge abstract because it does not necessarily impact patient care during hospitalisation.

In conclusion, our study highlights, on a national scale, that patients with CRDs, including COPD, asthma and CRF, were under-represented among hospitalised COVID-19 patients compared with hospitalised influenza patients. Nevertheless, we also confirmed that patients with respiratory diseases were at risk of developing severe COVID-19 and had a high mortality rate. The low proportion of patients with respiratory conditions found among the COVID-19 population could potentially be because vulnerable individuals followed the lockdown rules more stringently. We must therefore bear in mind that these patients are at risk of severe disease and not drop our guard too fast, even if only few of them were hospitalised during the first wave of COVID-19. Influenza and SARS-CoV-2 vaccination will be a key public health priority for these patients.

Acknowledgements: The authors thank Suzanne Rankin (Delegation for Clinical Research and Innovation (DRCI) University Hospital, Dijon, France) for reviewing the English.

Author contributions: G. Beltramo, P. Bonniaud, L. Piroth, C. Quantin and P. Tubert-Bitter were involved in the conception and design of the study. C. Quantin was the coordinator of the study. J. Cottenet, A-S. Mariet and C. Quantin were responsible for the data collection. G. Beltramo wrote the first draft. J. Cottenet was in charge of the analysis. J. Cottenet and C. Quantin accessed and verified the data. G. Beltramo, P. Bonniaud, M. Georges, 
L. Piroth, C. Quantin, P. Tubert-Bitter, A-S. Mariet and J. Cottenet were involved in the interpretation, critically reviewed the first draft, and approved the final version.

Conflict of interest: G. Beltramo has nothing to disclose. J. Cottenet has nothing to disclose. A-S. Mariet has nothing to disclose. M. Georges has nothing to disclose. L. Piroth has nothing to disclose. P. Tubert-Bitter has nothing to disclose. P. Bonniaud reports personal fees for advisory board work and other (travel expenses reimbursement) from Roche, personal fees for advisory board work and other (meeting attendance/registration) from Boehringer and Novartis, personal fees for advisory board work from Teva and AstraZeneca, other (travel expenses reimbursement) from Chiesi, other (meeting attendance/registration) from Stallergene, other (meeting attendance/registration and travel expenses reimbursement) from Sanofi, outside the submitted work. C. Quantin has nothing to disclose.

Support statement: The project was funded by the French National Research Agency.

\section{References}

1 Ahrenfeldt LJ, Nielsen CR, Möller S, et al. Burden and prevalence of risk factors for severe COVID-19 disease in the ageing European population - a SHARE-based analysis. Z Gesundh Wiss 2021; in press [https://doi.org/ 10.1007/s10389-021-01537-7].

2 Huang C, Wang Y, Li X, et al. Clinical features of patients infected with 2019 novel coronavirus in Wuhan, China. Lancet 2020; 395: 497-506.

3 Chung M, Bernheim A, Mei X, et al. CT Imaging features of 2019 novel coronavirus (2019-nCoV). Radiology 2020; 295: 202-207.

4 Poissy J, Goutay J, Caplan M, et al. Pulmonary embolism in patients with COVID-19: awareness of an increased prevalence. Circulation 2020; 142: 184-186.

5 Petersen E, Koopmans M, Go U, et al. Comparing SARS-CoV-2 with SARS-CoV and influenza pandemics. Lancet Infect Dis 2020; 20: e238-e244.

6 Burn E, You SC, Sena AG, et al. An international characterisation of patients hospitalised with COVID-19 and a comparison with those previously hospitalised with influenza. medRxiv 2020; preprint [https://doi.org/10. 1101/2020.04.22.20074336].

7 Barker WH, Mullooly JP. Pneumonia and influenza deaths during epidemics: implications for prevention. Arch Intern Med 1982; 142: 85.

8 Reacher M, Warne B, Reeve L, et al. Influenza-associated mortality in hospital care: a retrospective cohort study of risk factors and impact of oseltamivir in an English teaching hospital, 2016 to 2017. Euro Surveill Bull 2019; 24: 1900087.

9 Piroth L, Cottenet J, Mariet A-S, et al. Comparison of the characteristics, morbidity, and mortality of COVID-19 and seasonal influenza: a nationwide, population-based retrospective cohort study. Lancet Respir Med 2021; 9: 251-259.

10 Kwok S, Adam S, Ho JH, et al. Obesity: a critical risk factor in the COVID-19 pandemic. Clin Obes 2020; 10: e12403.

11 Apicella M, Campopiano MC, Mantuano M, et al. COVID-19 in people with diabetes: understanding the reasons for worse outcomes. Lancet Diabetes Endocrinol 2020; 8: 782-792.

12 Guan W-J, Liang W-H, Zhao Y, et al. Comorbidity and its impact on 1590 patients with COVID-19 in China: a nationwide analysis. Eur Respir J 2020; 55: 2001227.

13 Hazeldine J, Lord JM. Immunesenescence: a predisposing risk factor for the development of COVID-19? Front Immunol 2020; 11: 573662.

14 Halpin DMG, Faner R, Sibila O, et al. Do chronic respiratory diseases or their treatment affect the risk of SARS-CoV-2 infection? Lancet Respir Med 2020; 8: 436-438.

15 Grasselli G, Zangrillo A, Zanella A, et al. Baseline characteristics and outcomes of 1591 patients infected with SARS-CoV-2 admitted to ICUs of the Lombardy region, Italy. JAMA 2020; 323: 1574-1581.

16 Izquierdo JL, Almonacid C, González Y, et al. The impact of COVID-19 on patients with asthma. Eur Respir J 2021; 57: 2003142.

17 Liang EW, Edwin PC, Jing YT, et al. Reduction in asthma admissions during the COVID-19 pandemic: consequence of public health measures in Singapore. Eur Respir J 2021; 57: 2004493.

18 Richardson S, Hirsch JS, Narasimhan M, et al. Presenting characteristics, comorbidities, and outcomes among 5700 patients hospitalized with COVID-19 in the New York City Area. JAMA 2020; 323: 2052-2059.

19 Goyal P, Choi JJ, Pinheiro LC, et al. Clinical characteristics of Covid-19 in New York City. N Engl J Med 2020; 382: 2372-2374.

20 Walsh EE, Cox C, Falsey AR. Clinical features of influenza A virus infection in older hospitalized persons. J Am Geriatr Soc 2002; 50: 1498-1503.

21 Choi YJ, Park J-Y, Lee HS, et al. Effect of asthma and asthma medication on the prognosis of patients with COVID-19. Eur Respir J 2021; 57: 2002226. 
22 Lovinsky-Desir S, Deshpande DR, De A, et al. Asthma among hospitalized patients with COVID-19 and related outcomes. J Allergy Clin Immunol 2020; 146: 1027-1034.

23 Hughes-Visentin A, Paul ABM. Asthma and COVID-19: what do we know now. Clin Med Insights Circ Respir Pulm Med 2020; 14: 1179548420966242.

24 Cosgriff R, Ahern S, Bell SC, et al. A multinational report to characterise SARS-CoV-2 infection in people with cystic fibrosis. J Cyst Fibros 2020; 19: 355-358.

25 Colombo C, Burgel P-R, Gartner S, et al. Impact of COVID-19 on people with cystic fibrosis. Lancet Respir Med 2020; 8: e35-e36.

26 Halpin DMG, Singh D, Hadfield RM. Inhaled corticosteroids and COVID-19: a systematic review and clinical perspective. Eur Respir J 2020; 55; 2001009.

27 Le Quéré C, Jackson RB, Jones $\mathrm{MW}$, et al. Temporary reduction in daily global $\mathrm{CO}_{2}$ emissions during the COVID-19 forced confinement. Nat Clim Change 2020; 10: 647-653.

28 Guan W-J, Zheng X-Y, Chung KF, et al. Impact of air pollution on the burden of chronic respiratory diseases in China: time for urgent action. Lancet 2016; 388: 1939-1951.

29 Pozzer A, Dominici F, Haines A, et al. Regional and global contributions of air pollution to risk of death from COVID-19. Cardiovasc Res 2020; 116: 2247-2253.

30 D'Amato M, Molino A, Calabrese G, et al. The impact of cold on the respiratory tract and its consequences to respiratory health. Clin Transl Allergy 2018; 8: 20.

31 Bloom Cl, Drake $\mathrm{TM}$, Docherty $\mathrm{AB}$, et al. Risk of adverse outcomes in patients with underlying respiratory conditions admitted to hospital with COVID-19: a national, multicentre prospective cohort study using the ISARIC WHO Clinical Characterisation Protocol UK. Lancet Respir Med 2021; 9: 699-711.

32 Schultze A, Walker AJ, MacKenna B, et al. Risk of COVID-19-related death among patients with chronic obstructive pulmonary disease or asthma prescribed inhaled corticosteroids: an observational cohort study using the OpenSAFELY platform. Lancet Respir Med 2020; 8: 1106-1120.

33 Eger K, Bel EH. Asthma and COVID-19: do we finally have answers? Eur Respir J 2021; 57: 2004451.

34 Drake TM, Docherty AB, Harrison EM, et al. Outcome of hospitalization for COVID-19 in patients with interstitial lung disease: an international multicenter study. Am J Respir Crit Care Med 2020; 202: 1656-1665.

35 Guiot J, Henket M, Frix AN, et al. Single-center experience of patients with interstitial lung diseases during the early days of the COVID-19 pandemic. Respir Investig 2020; 58: 437-439.

36 Gaines J, Vgontzas AN, Fernandez-Mendoza J, et al. Obstructive sleep apnea and the metabolic syndrome: the road to clinically-meaningful phenotyping, improved prognosis, and personalized treatment. Sleep Med Rev 2018; 42: 211-219.

37 Cade BE, Dashti HS, Hassan SM, et al. Sleep apnea and COVID-19 mortality and hospitalization. Am J Respir Crit Care Med 2020; 202: 1462-1464.

38 Memtsoudis SG, Ivascu NS, Pryor KO, et al. Obesity as a risk factor for poor outcome in COVID-19-induced lung injury: the potential role of undiagnosed obstructive sleep apnoea. Br J Anaesth 2020; 125: e262-e263.

39 Verroken A, Scohy A, Gérard L, et al. Co-infections in COVID-19 critically ill and antibiotic management: a prospective cohort analysis. Crit Care 2020; 24: 410.

40 Bergman J, Ballin M, Nordström A, et al. Risk factors for COVID-19 diagnosis, hospitalization, and subsequent all-cause mortality in Sweden: a nationwide study. Eur J Epidemiol 2021; 36: 287-298.

41 Leung JM, Niikura M, Yang CWT, et al. COVID-19 and COPD. Eur Respir J 2020; 56: 2002108.

42 Alqahtani JS, Oyelade T, Aldhahir AM, et al. Prevalence, severity and mortality associated with COPD and smoking in patients with COVID-19: a rapid systematic review and meta-analysis. PLoS One 2020; 15: e0233147.

43 Zheng Z, Peng F, Xu B, et al. Risk factors of critical and mortal COVID-19 cases: a systematic literature review and meta-analysis. J Infect 2020; 81: e16-e25.

44 Khan MMA, Khan MN, Mustagir MG, et al. Effects of underlying morbidities on the occurrence of deaths in COVID-19 patients: a systematic review and meta-analysis. J Glob Health 2020; 10: 020503.

45 Tsakok MT, Qamhawi Z, Lumley SF, et al. COVID-19 CT pulmonary angiogram examinations and reported pulmonary embolism incidence: comparison between peak first wave and early second wave. Clin Radiol 2021; 76: 310-312. 